\title{
Limits on the role of retrieval cues in memory for actions: Enactment effects in the absence of object cues in the environment
}

\author{
Melanie C. Steffens \\ Friedrich-Schiller-Universität Jena, Jena, Germany \\ AXEl BuChNer \\ Heinrich-Heine-Universität Düsseldorf, Düsseldorf, Germany \\ AND \\ Karl F. Wender and Claudia Decker \\ Universität Trier, Trier, Germany
}

\begin{abstract}
Verb-object phrases (open the umbrella, knock on the table) are usually remembered better if they have been enacted during study (also called subject-performed tasks) than if they have merely been learned verbally (verbal tasks). This enactment effect is particularly pronounced for phrases for which the objects (table) are present as cues in the study and test contexts. In previous studies with retrieval cues for some phrases, the enactment effect in free recall for the other phrases has been surprisingly small or even nonexistent. The present study tested whether the often replicated enactment effect in free recall can be found if none of the phrases contains context cues. In Experiment 1, we tested, and corroborated, the suppression hypothesis: The enactment effect for a given type of phrase (marker phrases) is modified by the presence or absence of cues for the other phrases in the list (experimental phrases). Experiments 2 and 3 replicated the enactment effect for phrases without cues. Experiment 2 also showed that the presence of cues either at study or at test is sufficient for obtaining a suppression effect, and Experiment 3 showed that the enactment effect may disappear altogether if retrieval cues are very salient.
\end{abstract}

Remembering actions is prevalent in everyday life. Since the beginning of the 1980 s, a substantial number of studies have investigated memory for actions (for reviews, see Engelkamp, 1998; Steffens, 1998; Zimmer \& Cohen, 2001). Typically, memory performance has been compared using lists of verb-object phrases (e.g., point to the window) studied either by intentional verbal learning only (also referred to as verbal tasks; VTs) or by additional enactment (subject-performed tasks; SPTs). Verb-object phrases are enacted using body parts (scratch your head), objects present in the study context (knock on the table), or objects that are imagined (break the match). Most authors have agreed that enactment typically increases recall and recognition (e.g., Earles, 1996; Engelkamp, 1991; Nilsson \& Cohen, 1988; Nyberg, Nilsson, \& Bäckman, 1991). Interestingly, many languages even mirror this enactment effect: The word to grasp (or in German, begreifen) means understanding something particularly well. In spite of this agreement that enactment enhances recall in general, it has been found that the enactment effect in free recall is particularly pronounced for phrases with objects present in the study context, such as learning the phrase lift the mug in the presence of a coffee mug (e.g., Nyberg et al.,
1991; Steffens, Jelenec, Mecklenbräuker, \& Thompson, 2006). In fact, when phrases with objects were evaluated separately from phrases without objects in the same study list (e.g., insert the floppy disk was another phrase, and no floppy disk was present), phrases without objects were not recalled better in the enactment condition than in the verbal-learning condition (Steffens, Buchner, \& Wender, 2003). Since the presence of objects was counterbalanced, this could not have been due to an item effect. Given that the enactment effect has often been replicated, it is a surprising finding that it was limited to items with context cues present. As we will argue in detail below, it is still possible, if one evaluates closely each published study, that enactment does not increase recall in the absence of context cues. If there were no general enactment effect in free recall, this would seriously challenge most theories and models that have been proposed to account for the enactment effect. Alternatively, it could be that there is an enactment effect for phrases without objects unless it is suppressed by the presence of phrases with objects in the same study list. More generally, in this case, the enactment effect for a given set of phrases would depend on the list composition. In the light of efforts to isolate the fac-

M. C.Steffens, melanie.steffens@uni-jena.de 
tors that make particular action phrases memorable (R. L. Cohen, Peterson, \& Mantini Atkinson, 1987), this would also be an important finding. The main aim of the present research was to test between these two options.

Before we begin with a review of the differences between remembering actions and remembering verbal materials that have been identified, a general note is in order regarding the literature reviewed here. Strictly speaking, there are two different research traditions for the investigation of enactment effects. A substantial number of studies have been concerned with memory under ecologically valid conditions, where encoding is multimodal and rich. They have compared memory for phrases learned verbally without objects with memory for phrases enacted with objects (for a review, see Nilsson, 2000). These studies have typically yielded a recall advantage that we call an enactment plus object superiority effect. The combined effect of enactment plus object superiority is to increase recall over that for a verbal-learning condition (Engelkamp \& Zimmer, 1997, empirically isolated these two effects). The present article is concerned with the other research tradition: the question of how much merely carrying out a movement related to a verb-object phrase changes memory (see also von Essen \& Nilsson, 2003; Zimmer \& Engelkamp, 2003). Studies on the enactment plus object superiority effect are reviewed here only if they speak to this issue.

The studies introducing enactment have already indicated that it increases retention, as compared with verbal learning (R. L. Cohen, 1981; Engelkamp \& Krumnacker, 1980; Saltz \& Donnenwerth-Nolan, 1981). A number of published reports have shown consistent enactment effects in free recall without any exceptions (e.g., Earles \& Kersten, 2000, 2002; Earles, Kersten, Turner, \& McMullen, 1999; Engelkamp \& Jahn, 2003; Engelkamp, Seiler, \& Zimmer, 2004; Engelkamp \& Zimmer, 1996, 2002; Helstrup, 2004; Jahn \& Engelkamp, 2003; Knopf, 1995a; Koriat \& Pearlman-Avnion, 2003; Norris \& West, 1993; Ratner \& Hill, 1991; von Essen, 2005; Zimmer, 1991; Zimmer, Helstrup, \& Engelkamp, 2000). However, other studies have reported at least one exception - that is, one experiment or condition in which no enactment effect in free recall emerged (Brooks \& Gardiner, 1994; Daprati, Nico, Saimpont, Franck, \& Sirigu, 2005; Engelkamp, Mohr, \& Zimmer, 1991; Foley, Bouffard, Raag, \& DiSanto Rose, 1991; Helstrup, 1996, 2005; Helstrup \& Molander, 1996; Knopf, 1995b; Knopf, Mack, Lenel, \& Ferrante, 2005; Mohr, Engelkamp, \& Zimmer, 1989; Zimmer \& Engelkamp, 1985, 1989). In addition, direct comparisons have shown that the enactment effect is larger in cued recall than in free recall (Earles \& Kersten, 2000), and several findings have indicated that retrieval can even be hindered by enactment (Oloffson, 1996; Steffens et al., 2006, Experiment 1). Other limits of the enactment effect have been identified. For instance, enactment does not seem to benefit source memory (e.g., Hornstein \& Mulligan, 2004).

What processes can explain the fact that enactment increases retention under most circumstances, but not always? There is wide agreement that information pro- cessing during enactment is tied to the task demands of enactment (e.g., Engelkamp \& Dehn, 2000; Steffens et al., 2003). Carrying out actions, as compared with other encoding conditions, ensures the semantic processing of task-relevant features of verb-object phrases. In other words, enactment brings about, first, item-specific processing (Einstein \& Hunt, 1980), particularly of the verbs. This may be a reason why there is an enactment effect in free recall even for lists of verbs (throw, hit, etc.; see, e.g., Engelkamp, Zimmer, \& Kurbjuweit, 1995; Ratner \& Hill, 1991). A second reason why enactment improves memory is that not only the verb, but also the object and the verb-object relation are processed well during enactment, leading to an integrated or unitized memory representation (cf. Engelkamp, 1998; Kormi-Nouri, 1995; Saltz \& Donnenwerth-Nolan, 1981; Steffens, 1999; von Essen, 2005). This is why enactment effects are particularly pronounced in recognition and in cued recall with the object or verb as a cue.

In contrast to enhancing item-specific processing and integration, enactment does not generally enhance the processing of relations among successive to-be-learned action phrases (e.g., Engelkamp \& Zimmer, 1996, 2001, 2002; Koriat, Pearlman-Avnion, \& Ben Zur, 1998; Steffens, 1999; Steffens et al., 2006). Moreover, enactment does not increase item-context integration at a general level. For instance, memory for phrase-independent contexts (Baddeley, 1982) is not better for enacted than for observed phrases (Helstrup, 1989; Koriat, Ben Zur, \& Druch, 1991).

However, given that enactment enhances the processing of the verb-object relation, there is one type of context integration that should be increased by enactment (Steffens et al., 2003): When verb-object action phrases are studied with their objects present, the probability should be increased that these objects will receive attention, will be interactively encoded with the phrase, and can be used as retrieval cues (see also Nyberg et al., 1991; Steffens et al., 2006). For instance, if the phrase knock on the table is to be learned, participants in the enactment condition may knock on the table at which they are seated. Consequently, the phrase is interactively encoded with that table. During free recall, the table is a particularly powerful retrieval cue. It fits with this reasoning that the enactment effect in free recall is, indeed, much larger for the same action phrases in a condition with related objects present as cues in the environment than in a condition without these objects (Steffens et al., 2003). By using a multinomial model to decompose memory for action phrases into component processes, it was confirmed that the context integration advantage is due to superior retrieval of phrases with context objects (Steffens et al., 2006).

In the studies just mentioned, we routinely manipulated the presence only of such objects that were not very salient in the context. For instance, in a lab room, objects such as a pencil, a desk light, and so forth were present. The as-yetuntested assumption was that very salient objects would also be attended to and, thus, interactively encoded in a verbal-learning condition, increasing memory performance overall and eliminating the enactment effect. A hint that 
this assumption might be warranted was found when all the participants were instructed to use context objects that might aid recall (Steffens et al., 2003, Experiment 3): That instruction increased recall of phrases with context objects only in the verbal-learning condition (from 53\% to 68\%), but not in the enactment condition (in which correct recall dropped slightly from $81 \%$ to $80 \%$ ), and object presence no longer moderated the size of the enactment effect. An open question was whether the same pattern of findings would emerge if objects were very salient but not pointed out to the participants. We tested this in Experiment 3.

A surprising finding of our previous studies was that there was no statistically significant enactment effect in free recall for phrases without objects in the context (Steffens et al., 2003). On the basis of previous results and the theoretical consideration that enactment increases itemspecific processing, we had expected a smaller enactment effect for phrases without objects than for those with objects. Instead of a smaller one, the well-replicated enactment effect was not found at all for phrases without objects. There are two possible explanations for this. First, whereas there have been many demonstrations of enactment effects in free recall, almost all of the studies have used mixed lists that included phrases with imagined objects and phrases with the objects present, whether they were body parts or objects typically or incidentally present in the experimental environment (e.g., clap your hands, point to the door, clean the glasses), and the researchers have typically analyzed the enactment effect collapsed across all types of phrases (e.g., Earles \& Kersten, 2002; Norris \& West, 1993). Retrieval cues have thus often been present for at least a few phrases (e.g., von Essen, 2005). For example, in one of the studies that reported an enactment effect in free recall and appended the complete list of phrases (Earles et al., 1999), among the phrases used were wear scarf and look at watch. Similar objects can be found in other appendices (e.g., Engelkamp \& Jahn, 2003; Engelkamp \& Zimmer, 2002). Even when all the phrases contained imagined objects, retrieval cues can often be identified if materials are provided, such as file your fingernails (a phrase used by Brooks \& Gardiner, 1994) or put on the gloves (Jahn \& Engelkamp, 2003): Whereas these phrases contain imagined objects (file and glove), fingernails or hands were, of course, physically present and may have served as retrieval cues. Thus, it could be that there were retrieval cues present for several of the phrases in all those studies that have reported an enactment effect in free recall.

It is thus possible, even if it may appear unlikely, that the typically found enactment effect for verb-object phrases in free recall in between-subjects designs, averaged over the whole list of phrases, is due to a subset of phrases - those with retrieval cues. If this were so, virtually all theories and models of action memory would be seriously challenged. For instance, in an influential theory, Engelkamp (e.g., 1998) assumed that enactment adds the motor code generated during the execution of the action to an item representation in memory. This additional piece in the episodic representation is assumed to benefit retrieval at some later point in time, which is comparable to the dual-code theory of picture memory (Paivio, 1986). It is very difficult to see how such a theory would account for the finding that only a specific minority of the enacted phrases are recalled better than verbally learned phrases.

A second explanation for the finding of no enactment effect for phrases without objects could be a suppression effect: The presence of some phrases with objects might suppress the recall of phrases without objects, particularly in the enactment condition. For instance, if phrases with objects were recalled first, output interference could reduce recall of phrases without objects (Roediger \& Schmidt, 1980). If a suppression mechanism operated more strongly in the enactment condition, the enactment effect for phrases without objects would be suppressed. Put slightly differently, the availability of some object cues could turn the retrieval task into a cued-recall task in which performance benefits primarily from the verbobject associations formed during study.

\section{EXPERIMENT 1}

In Experiment 1, we tested the suppression hypothesisthat is, whether the presence of objects as cues for some phrases suppresses the enactment effect in recall for phrases without objects. In the condition in which cues were present for the experimental phrases, objects mentioned in the verb-object phrases were present in the experimental cubicle for office-related phrases (e.g., index cards or a joystick; henceforth, experimental phrases), and there were no objects that could serve as cues for food-related phrases (e.g., no sponge or no banana; henceforth, marker phrases). In the condition in which cues were not present for experimental phrases, the same number of objects was present as in the cues-present condition, but these objects were not related to any of the verb-object phrases (e.g., a cactus or a child's painting). The crucial prediction concerns the marker phrases. A suppression effect for marker phrases would be demonstrated if there were a typical enactment effect in the condition in which cues were not present for the experimental phrases, but not in the condition in which cues were present for the experimental phrases. In addition, we expected to replicate a larger enactment effect for experimental phrases in the cues-present condition than in the cues-not-present condition.

\section{Method}

Participants and Design. The participants were 84 students, 60 of them female, at the Universität Trier, who received credit or a small gift for participating. The design was a 2 (encoding condition: enactment vs. verbal learning) $\times 2$ (cue condition: cues present for experimental phrases vs. cues not present) $\times 2$ (type of action phrase: experimental phrases vs. marker phrases) mixed factorial with repeated measures on the last factor. The dependent variable was the percentage of action phrases recalled. Given a Type I error probability of $\alpha=.05$ and $N=84$, a large effect of encoding condition $(f=.40$; cf. J. Cohen, 1977$)$ could be detected with a probability of $1-\beta=.95$ (Faul, Erdfelder, Lang, \& Buchner, 2007).

Materials. A total of 40 verb-object phrases were selected that were taken from six different categories. The 14 experimental phrases were from the desk and computer categories (or from a common office category). It was rather to be expected that objects from these two categories would be in the room, which was desirable in order to keep the objects relatively nonsalient. Parallel to those, 14 
marker phrases (without objects as cues in any condition) were also chosen from two related categories, eating and kitchen (or from a common category food). Seven filler phrases (from the gardening category) were added for keeping total list length comparable to that in previous experiments (the conclusions remain identical if these fillers are included in the analyses below). As a recency buffer, five phrases were presented from the transportation category. Buffer phrases were excluded from all analyses. All the phrases are listed in the Appendix. The list structure was supported in a prestudy in which all the participants were perfectly able to sort the phrases into the correct categories.

The experiment was carried out in a lab with four cubicles. In two of them (cues-present condition), the 14 objects from the experimental phrases were present, so that these objects could be interactively encoded with the phrases and could serve as cues later on. In the other two cubicles (cues-not-present condition), 14 unrelated objects were presented that were assumed not to appear out of place (cactus, child's painting, etc.). There were no objects in any cubicle that could be related to the marker phrases. Cubicles were arranged so that the participants could not see the objects arranged in the other cubicles when entering the lab.

Procedure. The experiment took about $20 \mathrm{~min}$. The procedure largely followed that of previous experiments from our laboratory (Steffens et al., 2003). The participants were seated in experimental cubicles equipped with iMacs and were randomly assigned to the experimental conditions, with the restriction of arriving at equal $n \mathrm{~s}$ in all conditions. All the participants were informed that short phrases, each consisting of one verb and one object, would be presented on the computer screen, one after another, and that they should try to keep these phrases in mind. The participants in the enactment condition were additionally told to enact the phrases carefully while remaining seated - that is, to pretend to carry out each action. One phrase was presented for practice. After that, the experimenter reminded the participants of enactment if they had not moved properly. All the objects were placed just out of reach of the participants, and an experimenter oversaw all cubicles and made sure that the participants enacted all phrases without touching the objects. The instructions made no reference to the objects. Each verb-object phrase remained on the computer screen for $6 \mathrm{sec}$, with an interstimulus interval of $1 \mathrm{sec}$. Phrases were presented to the participants in an individually randomized order, with the restriction that experimental phrases, marker phrases, and fillers were distributed equally across the study list (in order to exclude possible confounds of serial position; cf. Zimmer et al., 2000): In the first five list positions, the second five, and so forth, there were two experimental phrases, two marker phrases, and one filler. After the study phase, there was a 5 -min written free recall test. Then, the participants were thanked, debriefed, and dismissed.

\section{Results}

As is common practice, action phrases were counted as recalled correctly if the reported words were identical to or synonymous with the study words. For all statistical tests, the Type I error level was set at $\alpha=.05$. Partial $R^{2}$ is reported as an indicator of the effect size (J. Cohen, 1977).

Overall enactment effect. In most studies that have reported an enactment effect, the data were collapsed across the entire list of verb-object phrases studied. If we followed this procedure, we would find the typically reported enactment effect replicated: In the enactment condition, participants recalled $46 \%$ of the study phrases, and in the verbal-learning condition, $35 \%$ were recalled, which was reflected in a main effect of encoding condition $\left[F(1,80)=15.43, R_{\mathrm{p}}^{2}=.16\right]$ in a 2 (encoding condition) $\times 2$ (cue condition) ANOVA. Overall, recall was only slightly better in the cues-present condition (42\%) than in the cues-not-present condition (39\%; all four $S E M \mathrm{~s}=2)$; thus, there was no main effect of cue condition. Also, there was no interaction effect (both of the other $F_{\mathrm{S}}<1.28$ ). In other words, it would appear again that enactment enhances recall in general. The finding that overall, recall is not much increased in the enactment, cues-present condition, as compared with the enactment, cues-not-present condition (which can be inferred from the absence of an interaction effect), may, however, be a first hint that participants do not recall more but do recall different action phrases. We tested this in the subsequent main analyses.

Recall of phrases with and without objects. Figure 1 shows that in the cues-present condition, the participants in the enactment condition recalled more of the experimental phrases than did those in the verbal-learning condition, which is equivalent to a large enactment effect for these phrases. In contrast, there is hardly any enactment effect for marker phrases. For the latter, there is, however, an enactment effect in the cues-not-present condition.

The statistical analyses corroborated these impressions. A 2 (encoding condition) $\times 2$ (cue condition) $\times 2$ (type of action phrase) ANOVA with repeated measures on the last factor and the percentage of phrases recalled as the dependent variable showed an interaction effect of all factors $\left[F(1,80)=4.62, R_{\mathrm{p}}^{2}=.06\right]$. Follow-up analyses were conducted that specifically tested our hypotheses. The

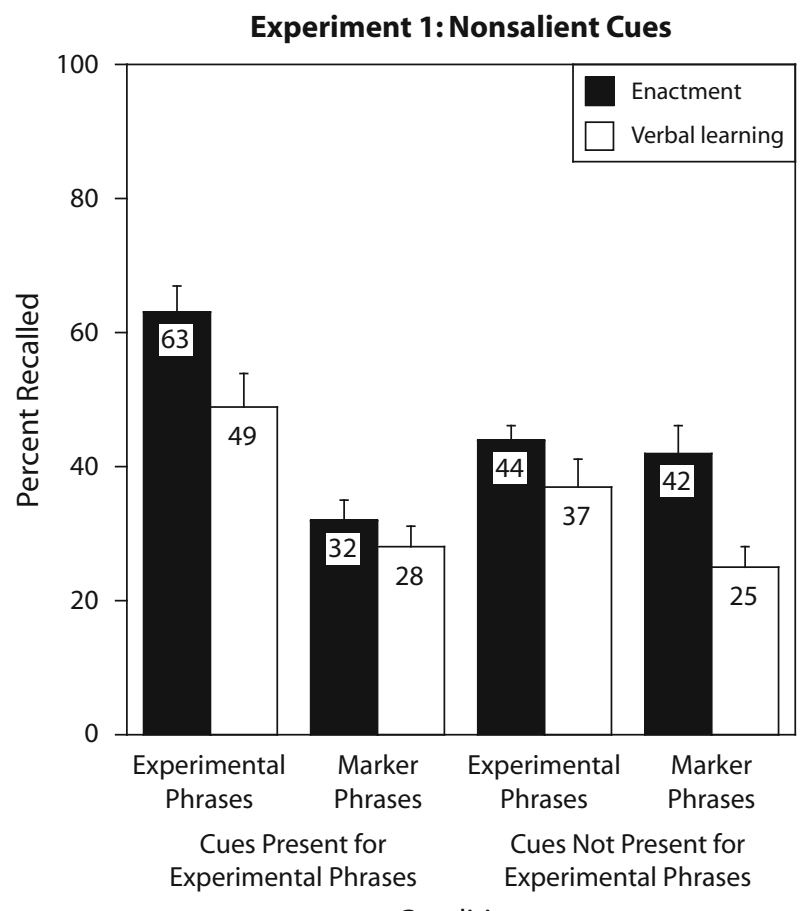

Condition

Figure 1. Percentage of action phrases recalled, separately for enactment and verbal learning, for experimental action phrases for which objects were present as cues in one condition but not in the other and for marker phrases that were identical in both conditions. Error bars represent the standard errors of the means. Experiment 1 comprised nonsalient cues: Experimental phrases were office-related; marker phrases were food-related. 
overall enactment effect in the cues-present condition that a subsequent ANOVA showed $\left[F(1,40)=4.91, R_{\mathrm{p}}^{2}=.11\right]$ was, as was expected, due to an enactment effect for experimental phrases - that is, phrases with objects present [simple main effect: $F(1,40)=4.92, R_{\mathrm{p}}^{2}=.11$ ]. For the marker phrases - those without objects - the effect was not significant [simple main effect: $F(1,40)<1, R_{\mathrm{p}}^{2}=$ $.02]$. A second follow-up ANOVA showed that there was also an overall enactment effect in the cues-not-present condition $\left[F(1,40)=9.15, R_{\mathrm{p}}^{2}=.19\right]$, but this time, the effect was statistically significant for the marker phrases [simple main effect: $\left.F(1,40)=10.53, R_{\mathrm{p}}^{2}=.21\right]$ but was not statistically significant for the experimental phrases [simple main effect: $F(1,40)<2.01, R_{\mathrm{p}}^{2}=.05$ ].

Supplementary analysis of output order. We analyzed output protocols in order to search for hints as to the processes underlying the suppression effect for marker phrases that we found. If output interference was responsible for the larger enactment effect for experimental phrases than for marker phrases in the cues-present condition, experimental phrases should be recalled earlier than marker phrases only in the cues-present condition. Neither the mean nor the median recall positions of types of phrases gave any hint that output interference was the basis of the suppression effect. Whereas experimental phrases in the cues-present condition were recalled later after verbal learning than after enactment [average percentiles were $.59, .52, .47$, and .54 , for experimental phrases after verbal learning and enactment and for marker phrases after verbal learning and enactment, respectively; $F(1,76)=5.93$, $\left.R_{\mathrm{p}}^{2}=.07\right]$, this effect did not interact with the cue condition $(F<1)$. Similarly, if only the beginnings of output protocols were analyzed (first 10 phrases), so that absolute, instead of relative, output positions could be evaluated, no interaction effect was found $(F<1)$.

\section{Discussion}

We considered two explanations of the previous finding that there was no enactment effect in free recall for phrases without objects present in the context if such objects were present for other phrases in the same study list. First, it could be that there is generally no enactment effect for phrases without objects - a finding seriously challenging virtually all theories and models of action memory, because they rest on the superiority of memory for actions over memory for verbal materials. A second possible explanation for the finding that there was no enactment effect for phrases without objects is a suppression effect: The enactment effect for phrases without objects can be suppressed by the presence of phrases with objects. Experiment 1 yielded evidence for suppression. The enactment effect for phrases without objects was eliminated when phrases with objects as cues were on the same list. In contrast, the enactment effect for phrases without objects was large in the same list when the objects cuing the other phrases were removed. In other words, when the objects that were mentioned in the experimental phrases were present, the enactment effect for the marker phrases disappeared. Thus, Experiment 1 demonstrated one limit on the role of retrieval cues in memory for actions: Ob- jects as retrieval cues appear to determine the enactment effect in free recall, but that effect is also obtained in the absence of objects as cues.

\section{EXPERIMENT 2}

The main aim of Experiment 2 was to test whether the presence of cues at study or at test is sufficient for suppressing the enactment effect for marker phrases. Half of the participants in Experiment 2 were in conditions that replicated those in Experiment 1 (cues were present for experimental phrases at study and test, as opposed to no cues being present at all). The other half of the participants had cues present for experimental phrases either only at study or only at test. Several studies have demonstrated that enactment effects remain unchanged if participants are blindfolded or asked to close their eyes at test (R. L. Cohen et al., 1987; Mulligan \& Hornstein, 2003). Similarly, other studies have shown no effect of retrieval context on action memory (Earles et al., 1999; Phillips \& Kausler, 1992). These findings seem to fit nicely with the conclusion that retrieval enactment often does not improve memory after enactment (Engelkamp, 1997): Apparently, not much context reinstatement is needed for successful recall after enactment. These findings suggest that the test context plays a minor role after enactment. Thus, we expected that the suppression effect for marker phrases would be replicated if cues for experimental phrases were present only at study. The predictions for cues present only at test were less clear. Possibly, cues at test can also be used more efficiently in an enactment condition than in a verbal-learning condition. One reason for this could be that verb-object phrases are better integrated after enactment, so the object can serve as a cue for the whole phrase.

Two additional major changes were introduced. First, which phrases were experimental and which were marker phrases was counterbalanced: In two cubicles, there were computer-related objects; in the other two, there were desk-related objects. Thus, for the participants in Cubicles 1 and 2, computer-related action phrases were experimental phrases, and desk-related action phrases were marker phrases. This was reversed in Cubicles 3 and 4. In addition, there were four cubicles that contained objects unrelated to the action phrases. These cubicles were used in the conditions in which the study phase, the test phase, or both had no objects present. Finally, instead of a recency buffer that contained action phrases, a delay was introduced between the study and the test phases. We did this in order to increase the generality of our findings in the light of previous findings that enactment particularly extends the recency effect (Zimmer et al., 2000).

\section{Method}

Participants and Design. The participants were 76 students, 21 of them male, at the Friedrich-Schiller-Universität Jena, who received credit and a small gift for participating. The design was a 2 (encoding condition: enactment vs. verbal learning) $\times 2$ (study cue condition: cues present for experimental phrases at study vs. cues not present) $\times 2$ (test cue condition: cues present for experimental phrases at test vs. cues not present) $\times 2$ (type of action phrase: experimental phrases vs. marker phrases) mixed factorial 
with repeated measures on the last factor. Which phrases were experimental and which were marker phrases was counterbalanced across participants.

Materials. A total of 30 verb-object phrases were selected that were taken from four categories. Most of the phrases were identical to those used in Experiment 1 (see the Appendix). The 20 experimental and marker phrases were from the desk and computer categories. Ten filler phrases (from the kitchen and gardening categories) were added.

The experiment was carried out in two adjacent labs, each with four cubicles. In four of them, either the 10 computer-related objects or the 10 desk-related objects were present (experimental phrases). In the other four cubicles, 10 unrelated objects were present.

Procedure. With the following exceptions, the procedure was identical to that in Experiment 1. The experiment took about $40 \mathrm{~min}$. The participants were seated in experimental cubicles equipped with iBooks. Experimental phrases, marker phrases, and fillers were distributed equally across the study list: In the first three list positions, the second three, and so forth, there was one phrase of each type. After the study phase, there was a delay of around 15 min with an unrelated filler task. After the subsequent 4-min written free recall test, the participants were thanked, debriefed, and dismissed.

\section{Results}

Overall enactment effect. In the enactment condition, the participants recalled $39 \%$ of the study phrases, and in the verbal learning condition, $31 \%$ were recalled, which was reflected in a main effect of encoding condition $\left[F(1,68)=9.99, R_{\mathrm{p}}^{2}=.13\right]$ in a 2 (encoding condition) $\times$ 2 (study cue condition) $\times 2$ (test cue condition) ANOVA. The only other effect in this analysis (all other $F_{\mathrm{S}}<2.32$ ) was an interaction of study and test cues $[F(1,68)=5.04$, $\left.R_{\mathrm{p}}^{2}=.07\right]$, reflecting that recall without cues at study or test ( $M=30 \%$ ) was inferior to recall with cues present at study, at test, or both (Ms $=38 \%, 36 \%$, and 33\%, respectively).

Recall of phrases with and without objects. Half of the participants in Experiment 2 were in conditions that replicated those in Experiment 1 (cues present for experimental phrases at study and test vs. no cues present at all). These conditions are presented in the upper part of Figure 2. The other half of the participants had cues present for experimental phrases either at study only or at test only (see the lower part of Figure 2). Descriptively, the enactment effect in the cues-present condition was larger for experimental than for marker phrases, whereas it was similar for experimental and marker phrases in the cues-not-present condition. This replicated the pattern found in Experiment 1 (even though the suppression effect for marker phrases in the cues-present condition was weaker than expected). As the lower part of Figure 2 shows, the data pattern in the cues-present condition was replicated when cues were present only at study: There was an enactment effect for experimental phrases that was suppressed for marker phrases. A similar but weaker pattern can be observed if cues are present only at test.

Given the large number of cells, the power to detect a nonorthogonal statistical interaction was quite limited. Therefore, we first carried out planned contrasts that captured our hypothesis (see Table 1). A suppression effect was computed in each experimental condition: the difference between the numbers of recalled experimental
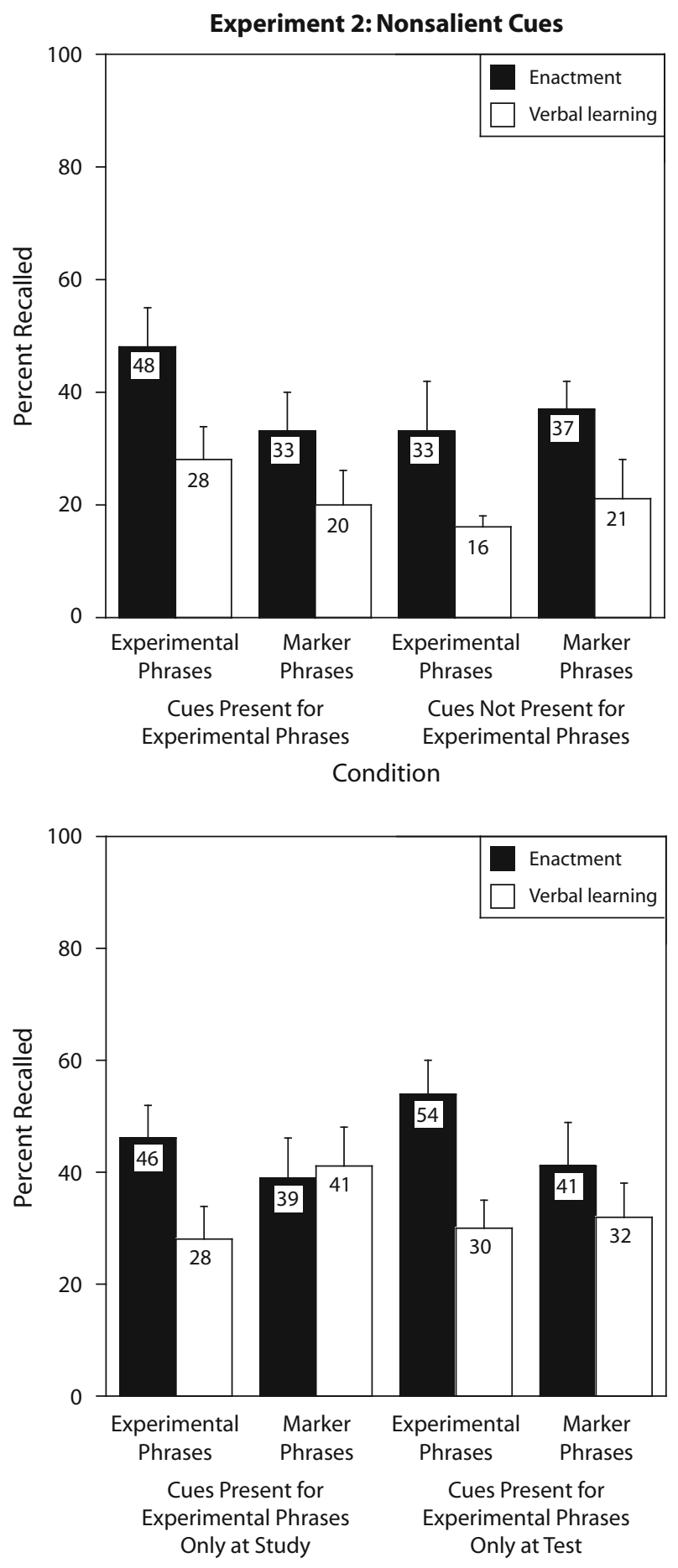

Figure 2. Percentage of action phrases recalled, separately for enactment and verbal learning, for experimental action phrases for which objects were present as cues at study and test (upper part, left), at study only (lower part, left), at test only (lower part, right), or not at all (upper part, right) and for marker phrases that were identical in both conditions. Error bars represent the standard errors of the means. Experiment 2 comprised nonsalient cues, and materials (office-related phrases) were balanced over phrase types. 
Table 1

Planned Contrasts Between Conditions Tested in Experiment 2, With Findings

\begin{tabular}{|c|c|c|c|c|c|c|c|c|c|c|}
\hline \multicolumn{8}{|c|}{ Experimental Condition } & \multirow[b]{4}{*}{ Diff } & \multirow[b]{4}{*}{$S E$} & \multirow[b]{4}{*}{$p$} \\
\hline \multicolumn{4}{|c|}{ Enactment } & \multicolumn{4}{|c|}{ Verbal Learning } & & & \\
\hline \multicolumn{2}{|c|}{ Study Cues } & \multicolumn{2}{|c|}{ No Study Cues } & \multicolumn{2}{|c|}{ Study Cues } & \multicolumn{2}{|c|}{ No Study Cues } & & & \\
\hline $\begin{array}{l}\text { Test } \\
\text { Cues }\end{array}$ & $\begin{array}{c}\text { No } \\
\text { Test } \\
\text { Cues }\end{array}$ & $\begin{array}{c}\text { Test } \\
\text { Cues }\end{array}$ & $\begin{array}{c}\text { No } \\
\text { Test } \\
\text { Cues }\end{array}$ & $\begin{array}{c}\text { Test } \\
\text { Cues }\end{array}$ & $\begin{array}{c}\text { No } \\
\text { Test } \\
\text { Cues }\end{array}$ & $\begin{array}{c}\text { Test } \\
\text { Cues }\end{array}$ & $\begin{array}{c}\text { No } \\
\text { Test } \\
\text { Cues }\end{array}$ & & & \\
\hline $\begin{array}{l}5 \\
\end{array}$ & 5 & 5 & -3 & -3 & -3 & -3 & -3 & .21 & .11 & $<.05$ \\
\hline 3 & 3 & -1 & -1 & -1 & -1 & -1 & -1 & .07 & .05 & $\mathrm{n} . \mathrm{s}$ \\
\hline 3 & -1 & 3 & -1 & -1 & -1 & -1 & -1 & .09 & .05 & .08 \\
\hline 1 & 1 & 1 & 1 & 1 & 1 & -3 & -3 & .05 & .05 & n.s \\
\hline
\end{tabular}

Note-Diff, difference estimate (estimate - hypothesized); $S E$, standard error of difference estimate.

phrases and marker phrases. Most important, according to the first contrast, there was more suppression in the three enactment conditions with cues (at study, at test, or both) than in the other five conditions (enactment with no cues present or any of the verbal-learning conditions). The second and third contrasts do not support the hypothesis that the suppression effect is restricted to study cues or to test cues. Finally, we found no general suppression effect across both encoding conditions.

In order to test our main hypotheses in detail, we then analyzed separately in which conditions the pattern of simple main effects in Experiment 1 was replicated. The overall enactment effect in the cues-present condition that an ANOVA showed $\left[F(1,20)=7.15, R_{\mathrm{p}}^{2}=.26\right]$ was, as was expected, due to an enactment effect for experimental phrases - that is, phrases with objects present [simple main effect: $\left.F(1,20)=4.26, R_{\mathrm{p}}^{2}=.18\right]$. For the marker phrases - those without objects - the enactment effect was not statistically significant [simple main effect: $F(1,20)<$ $2.36, p=.14]$. A second ANOVA showed that there was also an overall enactment effect in the cues-not-present condition $\left[F(1,15)=9.13, R_{\mathrm{p}}^{2}=.38\right]$, but this time, the effect was statistically significant for either type of phrase regarded separately in one-tailed tests [simple main effect for the marker phrases, $F(1,15)=3.37, \mathrm{p}=.09$ ]; for the experimental phrases, $F(1,15)=3.20, \mathrm{p}=.09]$. Thus, the pattern of findings in Experiment 1 was replicated, even though the suppression effect for marker phrases in the cues-present condition was less pronounced. However, that condition has been replicated several times before, and it is not crucial for the purposes of Experiment 2.

For the most interesting conditions in Experiment 2, a $2 \times 2 \times 2$ ANOVA showed an enactment effect and the expected interaction between type of phrase and encoding condition $\left[F(1,33)=3.08, R_{\mathrm{p}}^{2}=.09\right.$; statistically significant in a one-tailed test, $p<.09$; all other $F \mathrm{~s}<1.27]$. The simple main enactment effect was statistically significant for experimental phrases $\left[F(1,33)=13.64, R_{\mathrm{p}}^{2}=.30\right.$; also, regarded separately with cues only at study, $F(1,16)=$ $8.83, R_{\mathrm{p}}^{2}=.36$, and with cues only at test, $F(1,17)=6.36$, $R_{\mathrm{p}}^{2}=.27$ ], but there was none for marker phrases (either when both conditions were taken together or when they were analyzed separately; all $F_{\mathbf{S}}<1$ ). Thus, the presence of cues either at study or at test seems sufficient for suppressing the enactment effect for marker phrases.

\section{Discussion}

In sum, there are two main findings of Experiment 2. First, replicating Experiment 1, the enactment effect was observed in the absence of cues at study or at test. Second, Experiment 2 also replicated the suppression effect, even though in a weaker form than before: In the enactment condition, recall of phrases without cues was suppressed by the recall of phrases with cues. In addition, this effect held whether cues were present at study, at test, or both. We had expected that the presence of cues at study would be sufficient for obtaining a suppression effect: Mental reinstatement at test should allow the study cues to have their effect also in a different environment (see also Earles et al., 1999; Phillips \& Kausler, 1992). The finding that the presence of cues at test was also sufficient for obtaining a suppression effect was less expected. We will return to this in the General Discussion section.

A caveat with respect to Experiment 2 is that the design comprised many cells, so that the statistical power to detect differences between single cells was rather low (e.g., if one wanted to compare only the enactment conditions with cues at study only and those with cues at test only). Therefore, we will refrain from interpreting further any differences between single cells and will conclude that the presence of cues at study or at test appears to be sufficient for obtaining a suppression effect: If cues for a number of phrases are present at study or at test, the enactment effect for the other phrases is reduced. Objects present at study, at test, or at both seem to be used as retrieval cues to a lesser degree in a verbal-learning condition.

\section{EXPERIMENT 3}

The main aim of Experiment 3 was to replicate the enactment effect in a condition in which there were no objects present as cues for any phrases, because of its crucial meaning for action memory. We tested the generality of the finding by using a list of verb-object phrases that had no obvious taxonomic category structure. We were afraid, however, that there would be a second boundary condition on the role of retrieval cues in enactment effects. Specifically, we reasoned that with very salient objects (i.e., objects that clearly do not belong in an office but are related to the to-be-learned phrases), objects are used as cues in both the enactment and the verbal-learning conditions. To 
the degree that objects are so salient that they are used in both study conditions, there should be no enactment effect even for phrases with objects in the cues-present condition. In other words, whereas previous research, including Experiments 1 and 2, has shown that the enactment effect is especially pronounced for phrases with objects as cues, we thought that the enactment effect for those very phrases might also disappear.

\section{Method \\ Participants and Design. The participants were 64 students (50 of them female) at the Universität Trier, who received credit and a small gift for participating. None of them had participated in Ex- periment 1 . As in Experiment 1, the design was a 2 (encoding condi- tion: enactment vs. verbal learning) $\times 2$ (cue condition: cues present for experimental phrases vs. cues not present) $\times 2$ (type of action phrase: experimental phrases vs. marker phrases) mixed factorial with repeated measures on the last factor. Dependent variable was the percentage of action phrases recalled. \\ Materials and Procedure. The method followed that in Ex- periment 1, with one exception. A new list of 40 action phrases was compiled. Critical phrases were 28 phrases for which the presence of objects was manipulated in the cues-present condition. In Cubicle 1, 14 of the objects were present; in Cubicle 2, the other 14 were pres- ent. These objects were, at least in total, very salient (e.g., a lolly, a deck of cards, etc.; see the Appendix). Seven fillers were added. An additional 5 phrases served as a recency buffer. To reiterate briefly, a list of verb-object phrases was presented to a given participant either in the enactment or in the verbal-learning condition, followed by free recall. Types of action phrases were distributed evenly across the list. In the cues-present condition, there were objects that could serve as cues for the experimental phrases. In the cues-not-present condition, objects were present that could not serve as cues for any of the phrases. Cues for the marker phrases were never present. List composition (i.e., both the experimental and the marker phrases) was held constant across conditions.}

\section{Results}

Overall enactment effect. Parallel to the procedure in Experiments 1 and 2, in order to compare our findings with previous demonstrations of enactment effects, in a first analysis we disregarded the type of phrase. In the enactment condition, the participants recalled $42 \%$ of the study phrases; in the verbal-learning condition, 37\%. In contrast to the results in Experiment 1, overall recall was much better with objects as cues (47\%) than without $(32 \%$; all $S E M \mathrm{~s}=2)$, which is a first hint that the very salient objects indeed made the recall task easier. A 2 (encoding condition) $\times 2$ (cue condition) ANOVA yielded a main effect of cue condition only $\left[F(1,60)=23.35, R_{\mathrm{p}}^{2}=\right.$ .28]. The enactment effect was not statistically significant $(F=3.13, p=.08)$, and there was no interaction $(F<$ 2.18). The typical enactment effect was replicated, however, if the cues-not-present condition was analyzed separately $\left[F(1,30)=4.30, R_{\mathrm{p}}^{2}=.13\right]$.

Recall of phrases with and without objects. Most important, Figure 3 shows that in the cues-not-present condition, the enactment effect was replicated. In addition, it shows what we would expect if very salient objects can be used as cues in both the enactment and the verbal-learning conditions: In the cues-present condition, the participants in both the enactment and the verbal-learning conditions recalled most of the phrases with objects as cues.
The same ANOVA as that performed in Experiment 1 did not replicate that experiment's triple interaction effect $(F<1)$. Instead, it showed the expected interaction effect between cue condition and type of action phrase $[F(1,60)=$ $\left.136.09, R_{\mathrm{p}}^{2}=.69\right]$. Parallel to the procedure in Experiment 1 , we analyzed the cue conditions separately. In the cues-not-present condition, there was the expected enactment effect $\left[F(1,30)=6.03, R_{\mathrm{p}}^{2}=.17\right]$, no effect of type of action phrase $(F<2.52)$, and no interaction $(F<1)$. The only effect in the cues-present condition was a large effect of type of action phrase $\left[F(1,30)=197.02, R_{\mathrm{p}}^{2}=.87\right]$. There was no enactment effect $(F<1)$ and no interaction $(F<1.37)$. Even if experimental phrases, those with objects, were regarded separately, there was no statistically significant enactment effect (simple main effect: $F<1$ ).

\section{Discussion}

The central result of Experiment 3 is that it replicated the theoretically most important finding of Experiments 1 and 2: the enactment effect across all types of action phrases if there are no objects in the experimental cubicle that can be used as cues for any of the phrases. This finding points to the limited role of objects as retrieval cues for explaining the enactment effect. The second finding of Experiment 3 shows an additional limitation of those cues. The difference between Experiments 1 and 2, on the one

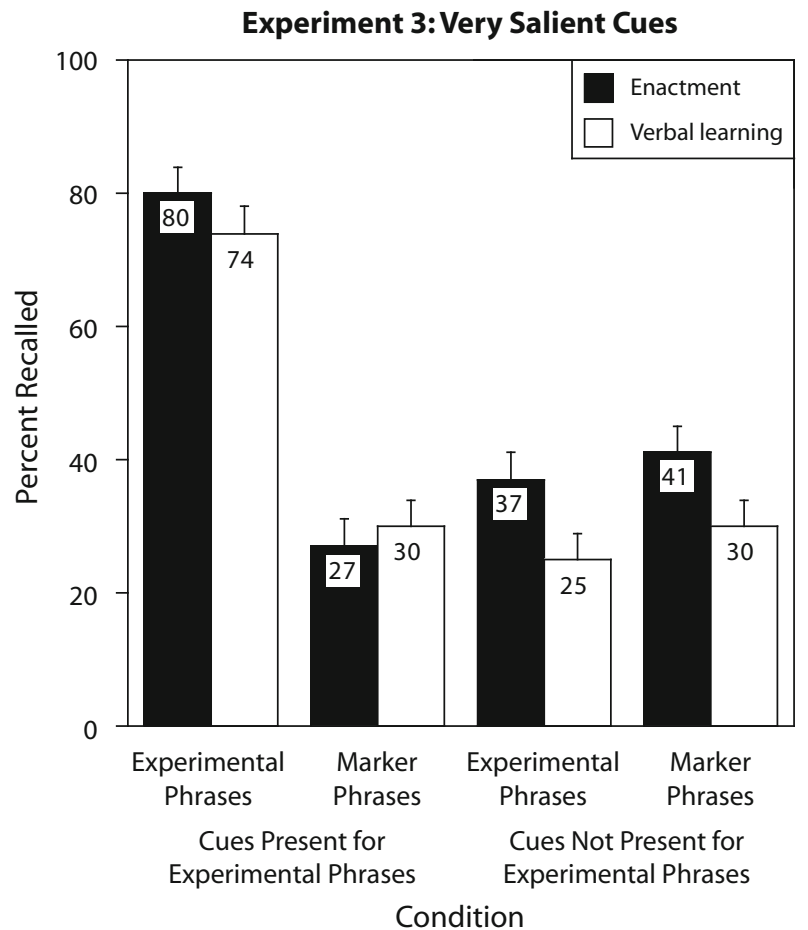

Figure 3. Percentage of action phrases recalled, separately for enactment and verbal learning, for experimental action phrases for which objects were present as cues in one condition but not in the other and for marker phrases that were identical in both conditions. Error bars represent the standard errors of the means. Experiment 3 comprised very salient cues; there was no obvious taxonomic list structure, and materials were balanced over phrase types. 
hand, and Experiment 3, on the other, was that the objects present in Experiments 1 and 2 fit nicely into the lab context (computer objects and desk objects), whereas those used in Experiment 3 were very salient. As was expected, these salient related objects were used as cues by the participants in both the enactment and the verbal-learning conditions. In fact, the participants in the verbal-learning condition were so successful at using the related objects as cues that the enactment effect was basically eliminated.

The latter aspect of the results must be considered with caution, however. First, with very salient objects, average recall was still $6 \%$ better in the enactment condition than in the verbal-learning condition. With more statistical power, a significant enactment effect might emerge in spite of object salience. Second, salience of objects was not directly manipulated. Instead, it differed between the cues-present conditions in Experiments 1, 2, and 3 (which also differed in list structure). Therefore, it is speculative to conclude that object salience was the crucial difference between the cues-present conditions in Experiments 1 and 2, in which an enactment effect was found, and the cues-present condition in Experiment 3, in which no statistically significant enactment effect emerged. A difference in object salience is very likely the cause of the difference in results between the experiments, given the increase in recall of cued phrases (e.g., from Experiment 1 to Experiment 3, by $50 \%$ in the verbal-learning condition), whereas recall of marker phrases was virtually identical in both experiments. Still, no firm conclusion should be drawn except for the modest modification of our formerly bold claim (Steffens et al., 2003) that objects as retrieval cues boost the enactment effect: Apparently, there are circumstances under which the presence of objects as cues rather reduces the enactment effect.

\section{GENERAL DISCUSSION}

In previous studies, we found a large enactment effect for action phrases with objects that could be used as interactive-context cues but no enactment effect for phrases without such objects. We now tested the hypothesis that there is never an enactment effect for phrases without objects present as cues in the experimental context against the suppression hypothesis - namely, that the enactment effect for phrases without objects is suppressed by the presence of phrases with objects. The data of Experiments 1-3 are consistent with the suppression hypothesis. The enactment effect for phrases without objects was eliminated only when phrases with objects as cues were on the same list. In contrast, the enactment effect for phrases without objects was large when the same phrases were on the list, but the objects were not present. Whereas Experiments 1 and 2 demonstrated the enactment effect for a categorized list of phrases without objects as cues, Experiment 3 replicated the enactment effect for an uncategorized list of phrases without such objects. Additional findings were that Experiment 1 replicated the previously found increased enactment effect for phrases with objects, Experiment 2 extended it to conditions in which objects are present at study only or at test only, and Experiment 3 showed that, with very salient objects, no statistically significant enactment effect may be observed.

The finding that the presence of objects for some phrases suppresses the enactment effect for the other phrases has several implications. First, the possibility existed that the presence of objects that serve as cues could be the crucial factor in producing the enactment effect in free recall. This is not the case. In mixed lists, consisting of phrases with and without objects, the objects indeed determine for which phrases there will be an enactment effect and for which there will be none. The situation is different in pure lists of phrases without objects. In such lists, an enactment effect in free recall was still found in Experiments 1-3. Thus, our findings show that cued items are recalled at the expense of noncued items. In Experiments 1 and 2, as well as in previous studies, this cuing effect was larger after enactment than after verbal learning. Consequently, the enactment effect for the noncued items was reduced or even eliminated. In contrast, we replicated the enactment effect in the no-cue conditions in all the experiments. Our suspicion that the enactment effect in free recall might have been overinterpreted before has thus been refuted empirically.

Second, this finding of an enactment effect in categorized and uncategorized lists of action phrases without context objects is reassuring for all theories, models, and mechanisms identified in research on action memory. As we demonstrated above, on the basis of previous research, the enactment effect in free recall could have been due to the presence of interactive-context cues. The crucial point with the present demonstration is that verb-object phrases in general, not a subset of these phrases with specific features, are better recalled after enactment than after verbal learning. In a nutshell, then, the present findings are in line with previous theorizing on enactment effects: Even if a confound was present routinely in most research on action memory, the theoretical implications of previous findings rest untouched.

A third implication of our findings relates them to research in which an attempt has been made to isolate the features of items that make them particularly memorable. We showed that the enactment effect for one group of action phrases - the phrases without cues - could be increased or decreased by changing the object cue context for another group of action phrases. Note that we did not even exchange those other phrases themselves. We simply manipulated whether related objects for those phrases were present or not. This was sufficient to change the enactment effect for the other phrases in the list. More generally, list composition may determine the size of memory effects for subsets of items. Consequently, it is futile to explore, with mixed lists of items, which of their features determine the size of an effect (e.g., Arar, Koliç, \& Molander, 1997; R. L. Cohen et al., 1987). A different list context alone may result in totally different memory effects.

Suppression effects have, of course, been demonstrated before in memory research, in the form of reduced recall for certain items due to the presence of other items in a given list, or in the form of increased recall for certain items only in the presence of other items. Consider, for in- 
stance, the generation effect (Slamecka \& Graf, 1978) that is, the finding that self-generated items (such as animal: ho--e) are remembered better than the same items would be if they were only read (animal: horse) (for a review, see Mulligan \& Lozito, 2004). It is known that the generation effect is boosted if the encoding task (generate vs. read) is manipulated within lists (Begg \& Snider, 1987; Slamecka \& Katsaiti, 1987). In fact, free recall generation effects in between-list designs have as often been absent or even reversed (for a review, see Steffens \& Erdfelder, 1998). In mixed lists, more attention is apparently devoted to generated, as opposed to read, items, so that the resulting generation effect can be regarded as an artifact of selectively displaced rehearsal that suppresses the recall of the read items. The experimental design (betweenvs. within-subjects manipulation of encoding task) has also been shown to modulate other memory effects-for instance, perceptual interference (Mulligan, 1999). The present findings go one step further, however. We showed that within the same encoding task, one item type can modify the memory effect for another item type. This finding is not entirely new, either. For instance, a generation effect was found for simple addition problems only when they were interspersed with multiplication problems (McNamara \& Healy, 1995).

We suggest that a process that is crucial to explaining these findings is attention allocation. To a certain degree, attention may be drawn to objects in the environment that may serve as retrieval cues "automatically." However, enactment directs attention to the related objects with a higher probability than verbal learning does. The relatedobject phrases are then interactively encoded with those objects with an increased probability, and the objects may serve as interactive-context cues or as primes. This is why related-object phrases had an enactment advantage in Experiment 1 . Note, however, that recall was far from perfect in the enactment condition, even given objects as cues. However, if objects are very salient because they seem totally out of place, as in Experiment 3, attention allocation to these objects is basically compulsory, so that there can hardly be an additional effect of enactment on attention allocation. In both cases, object-phrase associations can be formed during attending to the object pertaining to the to-be-learned phrase. These associations can support retrieval (see Eich, 1985, for basic research on interactive context cues). As the results of Experiment 2 show, the objects may also support recall in the enactment condition selectively, even if they are present at test only. We suggest that this effect is due to one or both of the following two mechanisms. First, given the particularly tight verbobject integration in the enactment condition, object cues at test may serve as potent recall cues. Second, objects at test could lead to an increased awareness in the enactment condition that all learned phrases could be carried out while sitting in the lab, hence restricting the search space for the to-be-recalled action phrases much as category names restrict the search space in verbal-learning experiments (Tulving \& Psotka, 1971).

An important point to notice is that, to the degree to which related objects are available during retrieval, the task becomes a cued recall rather than a free recall one. Again, participants' attention is drawn to the objects in the experimental context because these objects greatly aid the task at hand. Enactment effects on the to-be-learned phrases, such as increased distinctiveness of the verb, become irrelevant for the cued recall task, which relies heavily on the strength of the object-verb associations. The situation is different without external retrieval aids, where verb and object distinctiveness become important determinants of successful retrieval. This is why there is an enactment effect in situations in which the objects in the experimental context cannot function as retrieval cues - a characteristic of the cues-not-present conditions in all our experiments.

This explanation has the interesting implication that if participants' attention were not directed at the relatedobject cues at encoding, the memory task would be one of free recall, rather than cued recall, in which case an enactment effect should exist for phrases with and without objects. This was found in a recent study with young school-age children (Mecklenbräuker, Steffens, Jelenec, \& Goergens, 2007). In a first experiment, the effect of objects as cues was replicated with these children; in other words, the enactment effect was increased for phrases with objects present. In a second experiment, the procedure was slightly modified, in that the children were asked to keep in mind the action phrases in order to teach them to a green stuffed animal, Flocculi, who allegedly came from Mars and knew hardly anything about life on earth. As a result, the children visibly focused on Flocculi during the study and recall phases, rather than on the related-object cues. It turned out that the enactment effect for phrases with related-object cues did not exceed that for phrases without cues. These findings are in line with the hypothesis that the effect of related-object cues results from attention-based processes and the forming of objectphrase associations that are incidentally brought about by enactment.

By now, the related-objects effect has been found in a number of studies (Nyberg et al., 1991; Steffens et al., 2003; Mecklenbräuker et al., 2007; Steffens et al., 2006), so that it can be considered a stable phenomenon, on the one hand. On the other hand, the present research showed two important limitations on the role of retrieval cues in constituting the enactment effect. First, if, in a given list, there are no action phrases with objects present, there still is a reliable enactment effect in free recall. By implication, many previous demonstrations of enactment effects in free recall cannot be dismissed as being due simply to a subset of action phrases that were inadvertently cued by the presence of objects in the context. This is good news for the wealth of existing theories and models of action memory (e.g., Engelkamp, 1998). Second, if objects are very salient, they are exploited also in the verbal-learning condition, and the enactment effect disappears. Thus, objects as retrieval cues may determine whether the effects of enactment during encoding can be observed during retrieval, but enactment effects are demonstrated also in the absence of related objects that can be used as retrieval cues. 


\section{AUTHOR NOTE}

The research reported in this article was supported by Grant STE 938/5-1 from the Deutsche Forschungsgemeinschaft. We thank Beate Hoves and Tina Reicherter for help with the selection of materials and data collection and for fruitful discussions, and Rebekah E. Smith and Hubert Zimmer for valuable comments on an earlier draft. Correspondence concerning this article should be sent to M. C. Steffens, Institut für Psychologie, Friedrich-Schiller-Universität, Am Steiger 3, Haus 1, 07743 Jena, Germany (e-mail: melanie.steffens@uni-jena.de).

\section{REFERENCES}

Arar, L., Koliç, S., \& Molander, B. (1997, August). Familiarity, emotionality, motor activity, and memorability of imagined actions. Influence on the SPT effect. Paper presented at the Sixth European Workshop on Imagery and Cognition, Oslo.

Baddeley, A. D. (1982). Domains of recollection. Psychological Review, 89, 708-729.

BEGG, I., \& SNIDER, A. (1987). The generation effect: Evidence for generalized inhibition. Journal of Experimental Psychology: Learning, Memory, \& Cognition, 13, 553-563.

Brooks, B. M., \& GardineR, J. M. (1994). Age differences in memory for prospective compared with retrospective subject-performed tasks. Memory \& Cognition, 22, 27-33.

COHEN, J. (1977). Statistical power analysis for the behavioral sciences (Rev. ed.). Hillsdale, NJ: Erlbaum.

Cohen, R. L. (1981). On the generality of some memory laws. Scandinavian Journal of Psychology, 22, 267-281.

Cohen, R. L., Peterson, M., \& Mantini Atkinson, T. (1987). Interevent differences in event memory: Why are some events more recallable than others? Memory \& Cognition, 15, 109-118.

Daprati, E., Nico, D., Saimpont, A., Franck, N., \& Sirigu, A. (2005). Memory and action: An experimental study on normal subjects and schizophrenic patients. Neuropsychologia, 43, 281-293.

EARLES, J. L. (1996). Adult age differences in recall of performed and nonperformed items. Psychology \& Aging, 11, 638-648.

EARLeS, J. L., \& Kersten, A. W. (2000). Adult age differences in memory for verbs and nouns. Aging, Neuropsychology, \& Cognition, 7 , 130-139.

Earles, J. L., \& Kersten, A. W. (2002). Directed forgetting of actions by younger and older adults. Psychonomic Bulletin \& Review, 9 , 383-388.

Earles, J. L., Kersten, A. W., Turner, J. M., \& McMullen, J. (1999). Influences of age, performance, and item relatedness on verbatim and gist recall of verb-noun pairs. Journal of General Psychology, 126, 97-110.

EICH, E. (1985). Context, memory, and integrated item/context imagery. Journal of Experimental Psychology: Learning, Memory, \& Cognition, 11, 764-770.

EInSTEIN, G. O., \& Hunt, R. R. (1980). Levels of processing and organization: Additive effects of individual-item and relational processing. Journal of Experimental Psychology: Human Learning \& Memory, 6, 588-598.

EngelKamP, J. (1991). Memory of action events: Some implications for memory theory and for imagery. In C. Cornoldi \& M. A. McDaniel (Eds.), Imagery and cognition (pp. 183-219). New York: Springer.

ENGELKAMP, J. (1997). Memory for to-be-performed tasks versus memory for performed tasks. Memory \& Cognition, 25, 117-124.

EngelKamP, J. (1998). Memory for actions. Hove, U.K.: Psychology Press.

EngelKamp, J., \& Dehn, D. M. (2000). Item and order information in subject-performed tasks and experimenter-performed tasks. Journal of Experimental Psychology: Learning, Memory, \& Cognition, 26, 671-682.

ENGELKamp, J., \& JAHN, P. (2003). Lexical, conceptual and motor information in memory for action phrases: A multi-system account. Acta Psychologica, 113, 147-165.

EngelKamp, J., \& KRUMNACKer, H. (1980). Imaginale und motorische Prozesse beim Behalten verbalen Materials [Image and motor processes in the retention of verbal materials]. Zeitschrift für experimentelle und angewandte Psychologie, 27, 511-533.

Engelkamp, J., Mohr, G., \& Zimmer, H. D. (1991). Pair-relational encoding of performed nouns and verbs. Psychological Research, 53, 232-239.

Engelkamp, J., Seiler, K. H., \& Zimmer, H. D. (2004). Memory for actions: Item and relational information in categorized lists. Psychological Research, 9, 1-10.

ENGELKAMP, J., \& Zimmer, H. D. (1996). Organisation and recall in verbal tasks and in subject-performed tasks. European Journal of Cognitive Psychology, 8, 257-273.

ENGELKAMP, J., \& ZiMMER, H. D. (1997). Sensory factors in memory for subject-performed tasks. Acta Psychologica, 96, 43-60.

EngelKamp, J., \& Zimmer, H. (2001). Categorical and order information in free recall of action phrases. Psicologica, 22, 71-96.

ENGELKAMP, J., \& Zimmer, H. D. (2002). Free recall and organization as a function of varying relational encoding in action memory. Psychological Research, 66, 91-98.

EngelKamp, J., Zimmer, H. D., \& Kurbjuweit, A. (1995). Verb frequency and enactment in implicit and explicit memory. Psychological Research, 57, 242-249.

Faul, F., Erdfelder, E., Lang, A.-G., \& Buchner, A. (2007). G*Power 3: A flexible statistical power analysis program for the social, behavioral, and biomedical sciences. Behavior Research Methods, 39, 175-191.

Foley, M. A., Bouffard, V., RaAg, T., \& DiSanto Rose, M. (1991). The effects of enactive encoding, type of movement, and imagined perspective on memory of dance. Psychological Research, 53, 251-259.

Helstrup, T. (1989). Loci for act recall: Contextual influence on the processing of action events. Psychological Research, 51, 168-175.

Helstrup, T. (1996). What does it take for context to support action memory? Scandinavian Journal of Psychology, 37, 183-194.

HelstruP, T. (2004). The enactment effect is due to more than guesses and beliefs. Scandinavian Journal of Psychology, 45, 259-263.

HELSTRUP, T. (2005). In search of a motor element in memory for enacted events. European Journal of Cognitive Psychology, 17, 389-403.

Helstrup, T., \& Molander, B. (1996). Procedural dependence in action memory: Effects of verb form and individual vs group conditions. Scandinavian Journal of Psychology, 37, 329-337.

Hornstein, S. L., \& Mulligan, N. W. (2004). Memory for actions: Enactment and source memory. Psychonomic Bulletin \& Review, 11, 367-372.

Jahn, P., \& ENGELKamp, J. (2003). Design-effects in prospective and retrospective memory for actions. Experimental Psychology, 50, 4-15.

KNOPF, M. (1995a). Beyond verbal memory-Enhancing memory by acting. In B. J. Vellas, J. L. Albarede, \& P. J. Garry (Eds.), Mood and cognitive disorders: Facts and research in gerontology (pp. 43-53). New York: Springer.

KNOPF, M. (1995b). Das Erinnern eigener Handlungen im Alter [Memory for self-performed actions in old age]. Zeitschrift für Psychologie, 203, 335-349.

Knopf, M., Mack, W., Lenel, A., \& Ferrante, S. (2005). Memory for action events: Findings in neurological patients. Scandinavian Journal of Psychology, 46, 11-19.

Koriat, A., Ben Zur, H., \& Druch, A. (1991). The contextualization of input and output events in memory. Psychological Research, 53, 260-270.

Koriat, A., \& Pearlman-Avnion, S. (2003). Memory organization of action events and its relationship to memory performance. Journal of Experimental Psychology: General, 132, 435-454.

Koriat, A., Pearlman-Avnion, S., \& Ben Zur, H. (1998). The subjective organization of input and output events in memory. Psychological Research, 61, 295-307.

Kormi-Nouri, R. (1995). The nature of memory for action events: An episodic integration view. European Journal of Cognitive Psychology, 7, 337-363.

McNamara, D. S., \& Healy, A. F. (1995). A procedural explanation of the generation effect: The use of an operand retrieval strategy for multiplication and addition problems. Journal of Memory \& Language, 34, 399-416.

Mecklenbräuker, S., Steffens, M. C., Jelenec, P., \& Goergens, N. K. (2007). Retrieval cues in children's memory for actions: Attention-based processes brought about incidentally. Manuscript submitted for publication.

Mohr, G., Engelkamp, J., \& Zimmer, H. D. (1989). Recall and recognition of self-performed acts. Psychological Research, 51, 181-187. 
Mulligan, N. W. (1999). The effects of perceptual interference at encoding on organization and order: Investigating the roles of itemspecific and relational information. Journal of Experimental Psychology: Learning, Memory, \& Cognition, 25, 54-69.

Mulligan, N. W., \& Hornstein, S. L. (2003). Memory for actions: Self-performed tasks and the reenactment effect. Memory \& Cognition, 31, 412-421.

Mulligan, N. W., \& Lozito, J. P. (2004). Self-generation and memory. In B. H. Ross (Ed.), The psychology of learning and motivation: Advances in research and theory (Vol. 45, pp. 175-214). San Diego: Elsevier.

NiLsSON, L.-G. (2000). Remembering actions and words. In E. Tulving \& F. I. M. Craik (Eds.), The Oxford handbook of memory (pp. 137-148). Oxford: Oxford University Press.

Nilsson, L.-G., \& Cohen, R. L. (1988). Enrichment and generation in the recall of enacted and non-enacted instructions. In M. M. Gruneberg, P. E. Morris, \& R. N. Sykes (Eds.), Practical aspects of memory: Current research and issues. Vol. 1: Memory in everyday life (pp. 427-432). New York: Wiley.

Norris, M. P., \& West, R. L. (1993). Activity memory and aging: The role of motor retrieval and strategic processing. Psychology \& Aging, 8, 81-86.

Nyberg, L., Nilsson, L.-G., \& Bäckman, L. (1991). A component analysis of action events. Psychological Research, 53, 219-225.

Oloffson, U. (1996). The effect of enactment on memory for order. Psychological Research, 59, 75-79.

Paivio, A. (1986). Mental representations: A dual coding approach. New York: Oxford University Press.

Phillips, P. L., \& KAusler, D. H. (1992). Variation in external context and adult age differences in action memory. Experimental Aging Research, 18, 41-44.

Ratner, H. H., \& Hill, L. (1991). The development of children's action memory: When do actions speak louder than words? Psychological Research, 53, 195-202.

Roediger, H. L. III, \& SCHMidT, S. R. (1980). Output interference in the recall of categorized and paired-associate lists. Journal of Experimental Psychology: Human Learning \& Memory, 6, 91-105.

Saltz, E., \& Donnenwerth-Nolan, S. (1981). Does motoric imagery facilitate memory for sentences? A selective interference test. Journal of Verbal Learning \& Verbal Behavior, 20, 322-332.

Slamecka, N. J., \& Graf, P. (1978). The generation effect: Delineation of a phenomenon. Journal of Experimental Psychology: Human Learning \& Memory, 4, 592-604.

SlameckA, N. J., \& Katsaiti, L. T. (1987). The generation effect as an artifact of selective displaced rehearsal. Journal of Memory \& Language, 26, 589-607.

StefFens, M. C. (1998). Das Gedächtnis für ausgeführte Handlungen [Memory for performed actions]. Lengerich: Pabst.

StefFEns, M. C. (1999). The role of relational processing in memory for actions: A negative enactment effect in free recall. Quarterly Journal of Experimental Psychology, 52A, 877-903.

Steffens, M. C., Buchner, A., \& Wender, K. F. (2003). Quite ordinary retrieval cues may determine free recall of actions. Journal of Memory \& Language, 48, 399-415.

Steffens, M. C., \& ERdfelder, E. (1998). Determinants of positive and negative generation effects in free recall. Quarterly Journal of Experimental Psychology, 51A, 705-733.

Steffens, M. C., Jelenec, P., Mecklenbräuker, S., \& Thompson, E. M. (2006). Decomposing retrieval and integration in memory for actions: A multinomial modelling approach. Quarterly Journal of Experimental Psychology, 59, 557-576.

Tulving, E., \& PsotKa, J. (1971). Retroactive inhibition in free-recall: Inaccessibility of information available in the memory store. Journal of Experimental Psychology, 87, 1-8.

VON ESSEN, J. D. (2005). Enactment enhances integration between verb and noun, but not relational processing, in episodic memory. Scandinavian Journal of Psychology, 46, 315-321.

von Essen, J. D., \& Nilsson, L.-G. (2003). Memory effects of motor activation in subject-performed tasks and sign language. Psychonomic Bulletin \& Review, 10, 445-449.

Zimmer, H. D. (1991). Memory after motoric encoding in a generationrecognition model. Psychological Research, 53, 226-231.

Zimmer, H. D., \& Cohen, R. L. (2001). Remembering actions: A specific type of memory? In H. D. Zimmer, R. L. Cohen, M. J. Guynn, J. Engelkamp, R. Kormi Nouri, \& M. A. Foley (Eds.), Memory for action: A distinct form of episodic memory? (pp. 3-24). New York: Oxford University Press.

Zimmer, H. D., \& EngelKamp, J. (1985). An attempt to distinguish between kinematic and motor memory components. Acta Psychologica, 58, 81-106.

Zimmer, H. D., \& EngelKamp, J. (1989). Does motor encoding enhance relational information? Psychological Research, 51, 158-167.

Zimmer, H. D., \& EnGELKAMP, J. (2003). Signing enhances memory like performing actions. Psychonomic Bulletin \& Review, 10, 450-454.

Zimmer, H. D., Helstrup, T., \& Engelkamp, J. (2000). Pop-out into memory: A retrieval mechanism that is enhanced with the recall of subject-performed tasks. Journal of Experimental Psychology: Learning, Memory, \& Cognition, 26, 658-670.

\section{APPENDIX}

\section{Action Phrases Used in Experiment 1}

Experimental phrases: das Mousepad säubern*; die CD-ROM einlegen*; das Foto einscannen*; den Joystick bewegen; das Benutzerhandbuch lesen*; die Diskette beschriften*; den Drucker anschalten*; das Blatt zerknüllen; die Schreibtischlampe heranziehen*; den Papierkorb leeren*; die Schreibunterlage abwischen; den Bleistift anspitzen*; das Klebeband abreißen*; die Karteikarten sortieren* (clean the mouse pad; insert the CD-ROM; scan the photo; move the joystick; read the manual; label the floppy disk; switch on the printer; rumple the paper sheet; draw the desk light toward you; empty the waste paper basket; clean the blotting pad; sharpen the pencil; tear off the duct tape; sort the index cards)

Marker phrases: das Brot schmieren*; die Suppe würzen*; die Banane schälen*; den Brei umrühren; die Plätzchen ausstechen; die Möhre essen; den Saft trinken; die Kanne ausspülen; den Spülschwamm auswringen*; die Mikrowelle öffnen; den Teller abtrocknen; das Backblech herausziehen; das Silberbesteck polieren*; den Topf füllen (prepare a sandwich; spice the soup; peel the banana; stir the porridge; cut out the cookies; eat the carrot; drink the juice; clean the tea pot; wring the sponge; open the microwave; dry the plate; take out the baking tray; polish the silver; fill the pot)

Fillers: die Hecke schneiden*; den Salat pflanzen*; die Blume pflücken; den Rasen mähen*; das Unkraut ausrupfen; das Beet umgraben*; das Laub rechen* (cut the hedge; plant the lettuce; pick the flower; mow the lawn; tear out the weeds; dig up the bed; rake the leaves)

Recency buffer phrases: den Blinker setzen; den Helm aufsetzen; den Gurt anlegen; das Fahrrad lenken; die Handbremse lösen (use the turn signal; put on the helmet; put on the safety belt; steer the bike; undo the hand brake) 


\section{APPENDIX (Continued)}

\section{Action Phrases Used in Experiment 2}

Experimental phrases from Experiment 1 marked with a * were used as experimental and marker phrases in Experiment 2. Marker phrases and fillers in Experiment 1 with a * were used as fillers in Experiment 2.

Additional experimental phrases used in Experiment 2: den USB-Stick einstöpseln; die Kopfhörer aufsetzen; das Verbindungskabel hochheben; die Maus klicken; die Schreibunterlage glätten; die Tafel wischen; den Pappkarton treten; das Telefon bedienen; die Uhr stellen (plug in the USB stick; put on the headphones; lift the connecting cable; click the mouse; wipe the black board; kick the cardboard box; use the telephone; set the clock)

\section{Action Phrases Used in Experiment 3}

Experimental and marker phrases: die Praline auspacken; den Kaugummi kauen; den Schal umlegen; die Karten mischen; die Kerze anzünden; das Foto zerschneiden; den Kamm reinigen; die Serviette zerknüllen; den Joystick bewegen; die Schreibtischlampe heranziehen; den Kaktus gießen; den Kaffeebecher ausspülen; den Ball werfen; den Schlüsselanhänger befestigen; den Lolli lutschen; den Apfel schälen; den Handschuh anziehen; den Würfel fallenlassen; die Zigarette ausdrücken; das Hinweisschild lesen; den Aufkleber abziehen; den Schwamm ausdrücken; das Buch zuklappen; die Briefmarke ablecken; das Brillenetui öffnen; die Uhr stellen; die Kreide zerbrechen; das Telefon einstöpseln (open the praline; chew the gum; put on the scarf; shuffle the cards; light the candle; cut the photo; clean the comb; rumple the napkin; move the joystick; pull the desk light toward you; water the cactus; clean the coffee mug; throw the ball; fasten the key chain; suck the lollipop; peel the apple; put on the mitten; throw down the dice; stub out the cigarette; read the announcement; peel off the sticker; wring the sponge; close the book; lick the stamp; open the spectacle case; adjust the watch; break the chalk; plug in the phone)

Fillers: das Brot schmieren; das Kissen aufschütteln; das Paket aufreißen; die Geige stimmen; den Nagel einschlagen; den Stock durchbrechen; das Lasso werfen (prepare a sandwich; fluff the pillow; open the parcel; tune the violin; hammer in the nail; break the stick; throw the lasso)

Recency buffer phrases: den Hut aufsetzen; den Regenschirm aufspannen; die Seife ablegen; die Hecke schneiden; den Spiegel polieren (put on the hat; open the umbrella; put away the soap; cut the hedge; polish the mirror)

(Manuscript received August 24, 2006; revision accepted for publication April 20, 2007.) 